\author{
Article Type: Research Article $\quad$ Year: $2020 \quad$ V/I: 6(1) Pages: 32-41 \\ Corresponding Author: Selda Öz Soysal / seldaoz.oz@gmail.com \\ DOI: http://dx.doi.org/10.19148/ijhbs.713017 \\ Citation Information: \\ Öz Soysal, F. S. (2020). Validity and Reliability Study of the Negative Maintenance Scale to \\ the Turkish Young Adults. The International Journal of Human and Behavioral Science, 6(1), \\ 32-41. doi: 10.19148/ijhbs.713017
}

Received: 01/04/2020

Accepted: 15/06/2020

Published: 26/08/2020

\title{
Validity and Reliability Study of the Negative Maintenance Scale to the Turkish Young Adults
}

\begin{abstract}
The purpose of this work is to carry out the Turkish validity and reliability study of the Negative Maintenance Scale (NMS) developed by Dainton and Gross (2008). The research was conducted with a total of 505 students, 352 female and 153 male, who are first, second, third, and fourth year students enrolled in various departments of the Faculty of Education of Dokuz Eylül University. The six-factor structure of the scale was tested by confirmatory factor analysis. The obtained findings validated the six-factor structure of the NMS. The language validity of the scale was also examined. The reliability of the NMS was measured with test-retest reliability and internal consistency methods. Results indicated that the NMS is a valid and reliable measurement tool for assessing the maintenance strategies of negative relationships in young adults.
\end{abstract}

Keywords: negative, relationships, maintenance, confirmatory factor analysis, reliability

\section{Introduction}

Maintaining a relationship has been defined as behavioral dynamics that help preserve a relationship (Dindia, 2000, pp. 287-299). The maintenance of relationships is deemed as comprising intertwined behaviors in order to ensure the continuity of a valuable relationship, to prevent the weakening of a relationship, and to improve or repair and restore a relationship (Stafford, 1994). These behaviors are separated into two categories of routine and strategic (Dainton \& Stafford, 1993). Routine behaviors are behaviors involving daily interactions that implicitly serve the purpose of the relationship maintenance function without the intention of maintaining the relationship. However, strategic behaviors are behaviors that have a certain intention of maintaining the relationship (Dainton \& Strafford, 1993). According to Canary and Stafford (1992), relationship maintenance behaviors consist of five main behaviors. These are positivity (being joyful and positive behaviors towards the partner, not criticizing), openness (selfexpression and talking about the relationship), assurances (commitment, love, and loyalty), social networks (providing support and making the relationship more fun), and task-sharing (responsibilities for the continuance of the relationship) (Canary \& Stafford, 1992). Stafford, Dainton and Haas, (2000) later expanded these behaviors by adding two more. The first one of these behaviors is giving advice, defined as expressing the thoughts and feelings that partners have regarding each other. The second behavior is conflict management, which refers to the use of unifying behavior against conflict. 
Over the course of about 30 years, research has focused on maintaining relationships in different contexts such as marriage (Adams \& Baptist, 2012; Dainton, 2015; Ramirez, 2008; Weigel \& Ballard-Reisch, 2008), gay couples (Gutierrez, 2004; Haas \& Stafford, 2005; Ogolsky, 2009), friendships (Messman, Canary \& Hause, 2000; Oswald \& Clark, 2006), and sibling relationships (Myers \& Goodboy, 2010). In addition, the results of other research on the subject indicated that maintaining relationships strongly presumes important relationship features such as loyalty, relationship satisfaction, relationship stability, interest, and love (Canary, Stafford \& Semic, 2002; Ogolsky \& Bowers, 2013; Weiser \& Weigel, 2016). In studies that examined the relationships between attachment styles and relationship maintenance behaviors, there was a positive relationship between the attachment style and relationship-maintaining behaviors (Dainton, 2007), and there was a negative relationship between disinterested attachment, anxious attachment styles, and relationship-maintaining behaviors (Adams \& Baptist, 2012; Dainton, 2007).

However, not all relationship maintenance behaviors are positive social behaviors. Some antisocial behaviors may be functional for couples to maintain their relationships. Previous research results on the subject revealed that individuals use avoidance and antisocial strategies to maintain their relationships (Ayres, 1983; Dainton \& Stafford, 1993; Dindia \& Baxter, 1987; Guerro \& Chavez, 2005; Messman, Canary \& Hause, 2000). Ayres (1983) identified avoidance as one of these behaviors. Later, Dainton and Stafford (1993) determined that couples use avoidance and antisocial behavior strategies (lack of discussion, self-expression) to maintain romantic relationships. In addition, Messman, Canary, and Hause (2000) found that avoidance and indirect behaviors are used as two strategies by individuals in order to maintain their platonic friendships. Guerrero and Chavez (2005) determined that antisocial behavior is used to maintain opposite-sex friendships. Dainton and Gross (2008), as a result of their work with 188 undergraduate and graduate students, discovered that couples use 6 negative behaviors to maintain their relationships: jealousy induction (trying to make their partner jealous), avoidance (avoiding their partner or certain issues), destructive conflicts (starting a fight), allowing control (allowing the partner to plan or decide), spying (looking for specific information about their partners), and infidelity (having another relationship). The couples that maintain their relationships by using such behaviors usually report a lower level of liking, satisfaction, respect, mutual control, and fidelity (Goodboy, Meyers \& Communication Research Members, 2010).

Most of the research that concentrates on interpersonal relationships has focused on negative behaviors such as abuse, rape, destructive conflict, and cheating, but has neglected the fact that such behaviors are also used to maintain the relationship by some couples (Goodboy, Myers \& Communications Research Members, 2010). However, previous research did observe that couples use antisocial behaviors such as arguing, over-testing, and giving ultimatums to maintain their relationships (Baxter \& Dindia, 1990; Dindia \& Baxter, 1987).

The negative behaviors revealed by Dainton and Gross (2008) mean that individuals use these negative behaviors with the desire of maintaining their current relationships. Previous research indicated that jealousy can be used as a tactic of occupying the partner's mind (Buss, 1988). Destructive conflict beliefs are associated with self-centered or partner-centered relationship goals (Simon, Kobielski \& Martin, 2008). Spying can be a method of dealing with uncertainties in the relationship (Bevan \& Tidgewell, 2009). Avoidance of partners may increase partner satisfaction (Caughlin, 2002). Allowing oneself to be controlled by one's partner can allow the partner's need for interpersonal control to be met (Schutz, 1966). Infidelity, on the other hand, can help one of the partners fulfill their physical and emotional needs outside of the relationship (Roscoe, Cavanaugh \& Kennedy, 1988). When all these research results are considered together, it is observed that although these negative behaviors are labeled as antisocial behaviors, they also help maintain relationships. 
Dainton and Gross (2008) not only determined that there are inverse relationships between negative relationship maintenance behaviors and satisfaction. They also established that there is an inverse relationship between negative relationship maintenance behaviors and positive relationship maintenance behaviors. However, they found that negative relationship maintenance behaviors are a determinant of low-quality relationships.

Close relationships are influenced by the common beliefs, norms, and symbols of societies. Therefore, it is necessary to understand cultural foundations and values in order to comprehend relationship maintenance behaviors. Low-context cultures (e.g., Japan, China) place much greater importance on personal relationships and indirect communication exists more in these cultures than in higher-context cultures (e.g., Germany, Sweden). There are clear messages and strategies in high-context individualist cultures and these are used more than in collectivist cultures (Canary \& Yum, 2016, pp. 1-9). Considering all these points, it is important to examine the studies on relationship maintenance in different cultures.

There is no study in the related literature on relationship maintenance in Turkey, except for the adaptation of the Positive Relationship Maintenance Scale to Turkish culture (Öz Soysal, Uz Baş \& Aysan, 2019). It is therefore believed that it is important to introduce a measurement tool related to the maintenance of negative relationships in Turkey. The purpose of this work is to carry out the validity and reliability study of the Negative Maintenance Scale developed by Dainton and Gross (2008) and adapt it to Turkish.

\section{Method}

\subsection{Study Group}

The participants of the study consisted of first, second, third, and fourth year students from various departments of the Dokuz Eylül University Buca Faculty of Education, who were continuing their education and training in the 2019-2020 academic year. The ages of the participants ranged between 19 and 25, with 367 students (average age $=21.03, \mathrm{SD}=0.05)$, of which $352(69.7 \%)$ were female (average age $=20.08, \mathrm{SD}=1.3)$ and $153(30.3 \%)$ were male (average age $=21.17$, $\mathrm{SD}=1.25)$.

\subsection{Instrument}

\subsubsection{Negative Maintenance Scale}

The scale developed by Dainton and Gross (2008) consists of 20 items. Scale items are Likerttype and are scored between 1 (strongly disagree) and 7 (totally agree). The scale has six factors. These are jealousy induction (two items, e.g., "I flirt with others to make my partner jealous"), avoidance (four items, e.g., "I avoid matters that cause controversy"), spying (three items, e.g., "I check my partner's email and telephone messages"), infidelity (three items, e.g., "I flirt with others to avoid getting bored"), destructive conflict (four items, e.g., "I fight with my partner when I am sad"), and allowing control (five items, e.g., "I quit the activities I enjoyed because my partner did not like them") (Dainton \& Gross, 2008). The scale was applied to 151 people with an average age of $39.4(\mathrm{SD}=12.78)$. The results according to the Cronbach alpha coefficients of each individual factor were respectively found as 0.89 for jealousy, 0.86 for avoidance, 0.77 for spying, 0.80 for infidelity, 0.77 for destructive conflict, and 0.74 for allowing control (Dainton $\&$ Gross, 2008).

\subsection{Process}

First of all, permission was requested from the researchers who developed the scale in order to adapt it to the Turkish culture. Later, the scale was translated into Turkish by the researcher. These translations were reexamined by three faculty members who had at least a PhD in Psychological 
Counseling and Guidance and who had a good command of both their mother tongue and English. The items of the scale were rearranged in line with the feedback obtained. The scale was then redelivered to these faculty members for re-assessment. The scale was finalized by taking into account the final assessments of the faculty members and the compliance with the items. The adapted Turkish form and the original form of the scale were applied with 3-week intervals to 60 individuals, who were fourth year students of the Dokuz Eylül University Buca Faculty of Education, Department of English Teaching, in order to determine the language validity of the Negative Maintenance Scale (NMS). The scale was also reapplied to another group of 132 people with 2-week intervals in order to investigate the reliability of the scale and the reliability of test repetition.

It took about 10-15 minutes for students to complete the scale. The implementation was carried out by the researcher.

\subsection{Data Analysis}

The data obtained from the scales were transferred to SPSS 23.0 in order to conduct the reliability and validity analysis of the Turkish version of the NMS. Confirmatory factor analysis (CFA) was carried out in order to determine the validity of the structure of the scale. The AMOS 24 package program was used in the CFA analysis. The Cronbach alpha coefficient of each factor was calculated first in order to analyze the reliability of the scale. SPSS 23.0 was used to calculate the Cronbach alpha coefficient.

\section{Findings}

Before analyzing the validity and reliability of the NMS, some descriptive statistics were calculated, as shown in Table 1.

Table 1. Descriptive Statistics on the Overall and Individual Factor Scores of the Negative Maintenance Scale

\begin{tabular}{lcccccccc}
\hline Factors & & & & \multicolumn{2}{c}{ Skewness } & \multicolumn{2}{c}{ Kurtosis } \\
& $\mathbf{n}$ & $\overline{\mathrm{X}}$ & $\mathbf{S x}_{\mathbf{x}}$ & $\mathbf{S x}_{\mathbf{2}}{ }^{2}$ & Coefficient & Se & Coefficient & Se \\
\hline Jealousy & 505 & 5.5 & 0.1 & 0.01 & 1.1 & 0.1 & 0.5 & 0.2 \\
Avoidance & 505 & 16.3 & 0.2 & 0.04 & -0.2 & 0.1 & -1.2 & 0.2 \\
Spying & 505 & 9.9 & 0.2 & 0.04 & 0.7 & 0.1 & -1.0 & 0.2 \\
Infidelity & 505 & 9.1 & 0.1 & 0.01 & -0.2 & 0.1 & -1.0 & 0.2 \\
Destructive conflict & 505 & 12.9 & 0.2 & 0.04 & 1.1 & 0.1 & 0.7 & 0.2 \\
Allowing control & 505 & 21.1 & 0.2 & 5.29 & -0.5 & 0.1 & -1.1 & 0.2 \\
\hline
\end{tabular}

When Table 1 is examined, it is observed that the average scores of the scale vary between 5.5 and 21.08. The coefficients of skewness are between -0.2 and 1.1 and the coefficients of kurtosis are between -0.5 and -1.2. According to Tabachnic and Fidell (2015, pp. 78-82), the coefficients of skewness and kurtosis being \pm 1.5 indicates that the data are normally distributed. When the obtained values are examined, it is concluded that the data meet the normal distribution assumption.

\subsection{Language Validity}

The adapted Turkish form and the original form of the scale were applied with 3-week intervals to 52 individuals who were fourth year students of the Dokuz Eylül University Buca Faculty of Education, Department of English Teaching, in order to determine the language validity of the NMS. The correlation coefficient obtained as a result of the two applications was 0.83 . This indicates that the adapted form of the scale and the original form measure the same structure. 


\section{2. Structural Validity}

The original scale developed by Dainton and Gross (2008) has six factors. One of the aims of this study is to test the structural validity of the Turkish form of the original six-factor structure. Therefore, CFA was used and the first analyses were carried out to test the original six-factor structure.

Based on the data of 505 respondents, t-values and model-data fit indices were calculated to test the significance of the diagram and standardized factor loads and significance of factor loads for the six-factor model. The indexes showing the model-data fit for the six-factor structure of the scale are provided in Table 2.

Table 2. Values for Negative Maintenance Scale Goodness of Fit Indices

\begin{tabular}{ccccccc}
\hline$\chi^{2} /$ SD & NFI & IFI & CFI & GFI & AGFI & RMSEA \\
\hline 1.9 & 0.90 & 0.95 & 0.94 & 0.94 & 0.92 & 0.04 \\
\hline
\end{tabular}

The chi-square ( $\left.\chi^{2} / \mathrm{SD}\right)$ value of the fitness indices given in Table 2 being between 0 and 2 can be interpreted as evidence of the model being good (Schermelleh-Engel \& Moosbrugger, 2003). In this case, the calculated chi-square value $\left(\chi^{2} / \mathrm{SD}=3.6, \mathrm{p}<0.05\right)$ can be interpreted as the model having a good fitness level. On the other hand, fit indices of NFI, IFI, CFI, GFI, and AGFI being larger than or equal to 0.90 also indicates a good fitness level. If the value of the RMSEA index is less than or equal to 0.05 , it means good fitness, while values between 0.05 and 0.10 mean an acceptable fit (Haigh, Moore, Kashdan \& Fresco, 2011; Schermelleh-Engel \& Moosbrugger, 2003). According to this information, it can be stated that the model has a good fitness level according to the NFI (0.90), IFI (0.95), CFI (0.94), GFI (0.94), AGFI (0.92), and RMSEA (0.04) indices calculated related to the six-factor structure of the NMS. A diagram of the CFA applied within the scope of the NMS validity study is shown in Figure 1.

\subsection{Reliability of the NMS}

Reliability is defined as a measurement tool to give precise, consistent measurements that are also free of random errors (Yurdabakan, 2008, pp. 38-66). In the current study, the reliability of the NMS was examined by test repetition and Cronbach alpha internal consistency estimation methods. For this purpose, the scale was applied to a group of 132 individuals at 2-week intervals by providing the necessary conditions, and the test repetition reliability coefficient was calculated as $0.84(\mathrm{p}<0.01)$. Cronbach alpha reliability coefficients calculated according to the internal consistency estimation method of the scale were calculated separately for both the total scale and its six factors, and the results are presented in Table 3.

Table 3. Negative Maintenance Scale Reliability Coefficients

\begin{tabular}{lc}
\hline Factors & Internal Consistency Reliability Coefficient \\
\hline Jealousy & 0.72 \\
Avoidance & 0.70 \\
Spying & 0.82 \\
Infidelity & 0.83 \\
Destructive conflict & 0.78 \\
Allowing control & 0.74 \\
\hline
\end{tabular}




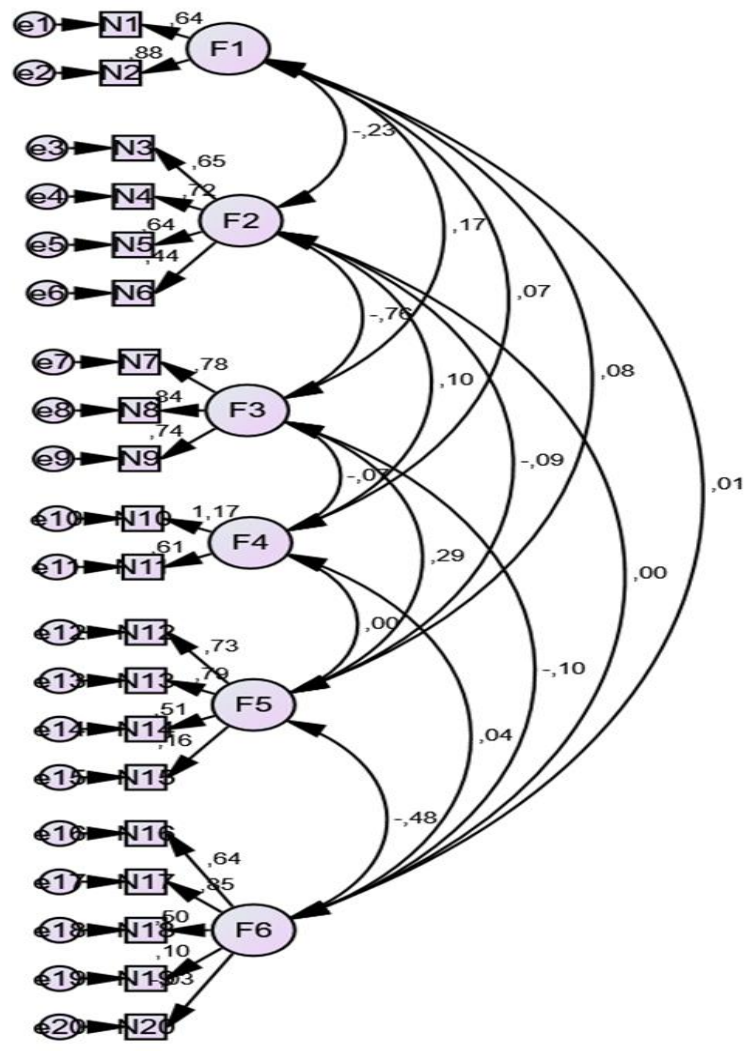

Figure 1. . Negative Maintenance Scale Path Diagram

\section{Discussion and Conclusion}

The purpose of this study was to establish the validity and reliability of the NMS developed by Dainton and Gross (2008) and to adapt it to Turkish. Structural validity and language validity studies were conducted in order to achieve this goal. Internal consistency and test-retest reliability coefficients were calculated to determine the reliability of the scale.

CFA was conducted in order to obtain evidence of the structural validity of the scale. As a result of this analysis, acceptable levels of model-data fit indices were obtained. The original six-factor structure of the scale was confirmed by Turkish students at the same time that the CFA was carried out. In the study conducted for the language validity of the scale, the coefficient obtained as a result of two applications was determined as 0.83 . This indicates that the adapted form of the scale and the original form measure the same structure.

The internal consistency coefficient of NMS, consisting of a total of 20 items, was calculated according to its six factors, yielding a coefficient of 0.72 for jealousy, 0.70 for avoidance, 0.82 for spying, 0.83 for infidelity, 0.78 for destructive conflict, and 0.7 for allowing control. The obtained values indicate that the scale has high internal consistency. In addition, the correlation coefficient of 0.84 obtained as a part of the test-retest study was evaluated as another indicator that the scale was reliable.

In this study, some psychometric characteristics of the NMS were examined and the findings showed that the tool is usable. However, this research has some limitations. The subjects included in the sample are only unmarried students studying in the Dokuz Eylül University Faculty of 
Education. In future studies, sample groups with different ages and different marital statuses in different provinces would help offer more information about the NMS.

The NMS can be used by psychological counselors and marriage and family counselors. It can contribute to experimental studies being carried out with individuals about maintaining relationships, especially in the pre-marital counseling process. At the same time, it can facilitate the examination of the purpose of the relations between subjects, as well as their attachment and communication styles. In addition, more research can be suggested on the most frequently used negative relationship maintenance behaviors in different types of relationships (such as sibling relationships or friendships).

\section{References}

Adams, R. D. \& Baptist, J. A. (2012). Relationship maintenance behavior and adult attachment: An analysis of the actor-partner interdependence model. The American Journal of Family Therapy, 40(3), 230-244. doi: 10.1080/01926187.2011.605047.

Ayres, J. (1983). Strategies to maintain relationship: Their identification and perceived usage. Communication Quarterly, 31, 62-67

Baxter, L., A. \& Dindia, K. (1990). Marital partners' perceptions of marital maintenance strategies, Journal of Social and Personal Relationships, 7(2), 187-208. doi: $10.1177 / 0265407590072003$

Bevan, J. L. \& Tidgewell, K. D. (2009). Relational uncertainty as a consequence of partner expressions. Communication Studies, 60, 305-323. doi: 10.1080=10510970902956057.

Buss, D. M. (1988). From vigilance to violence: Tactics of mate retention in American undergraduates. Ethology and Sociobiology, 9, 291-317. doi:10.1016=01623095(88)90010-6

Canary, D. J. \& Stafford, L. (1992). Relational maintenance strategies and equity in marriage. Communication Monographs, 59, 243-267. doi: 10.1080/ 03637759209376268

Canary, D. J, Stafford, L. \& Semic, B. (2002). A panel study of the associations between maintenance strategies and relational characteristics. Journal of Marriage and the Family, 64, 395-406.

Canary, D. J. \& Yum, Y.O. (2016). Relationship maintenance strategies. In book: The International Encyclopedia of Interpersonal Communication. (pp.19). doi:10.1002/9781118540190.wbeic0248

Caughlin, J. P. (2002). The demand withdraw pattern of communication as a predictor of marital 
satisfaction over time: Unresolved issues and future directions. Human Communication Research, 28, 49-85.

Dainton, M. \& Stafford, L. (1993). Routine maintenance behaviors: A comparison of relationship type, partner similarity and sex differences. Journal of Social and Personal Relationships, 10, 255-271.

Dainton, M. (2007). Attachment and marital maintenance. Communication Quarterly, 55(3), 283-298. doi: 10.1080/01463370701490083.

Dainton, M. \& Gross, J. (2008). The use of negative behaviors to maintain relationships. Communication Research Reports, 25, 179-191. doi: 10.1080/08824090802237600.

Dainton, M. (2015). An Interdependence approach to relationship maintenance in interracial marriage. Journal of Social Issues, 71(4), 772-787. doi: 10.1111/josi.12148.

Dindia, K. \& Baxter, L. A. (1987). Strategies for maintaining and repairing marital relationships. Journal of Social and Personal Relationships, 4, 143-158.

Dindia, K. (2000). Relational maintenance. In C. Hendrick and S. S. Hendrick (Eds.), Close relationships: A sourcebook (pp. 287-299). Thousand Oaks, CA: Sage.

Goodboy, A. K., Myers, S. A. \& Members of Investigating Communication. (2010). Relational quality indicators and love styles as predictors of negative maintenance behaviors in romantic relationships. Communication Reports, 23, 65-78.

Gutierrez, V. (2004). Maintenance behaviors and conflict level, areas, and resolution strategies in same-sex couples. Unpublished doctoral dissertation, University of California, California. USA .

Haas, S. M. \& Stafford, L. (2005). Maintenance behaviors in same-sex and marital relationships: A matched sample comparison. Journal of Family Communication, 5, 43-60. doi: 10.1207/s15327698jfc05013.

Haigh, E. A. P., Moore, M. T., Kashdan, T. B. \& Fresco, D. M. (2011). Examination of the factor structure and concurrent validity of the Langer Mindfulness/Mindlessness Scale. Assessment, 18(1), 11-26.

Messman, S. J., Canary, D. J. \& Hause, K. S. (2000). Motives to remain platonic equity, and the use of maintenance strategies in opposite-sex friendships. Journal of Social and Personal Relationships, 17, 67-94.

Merolla, A. J. (2012). Connecting here and there: A model of long-distance relationship maintenance. Personal Relationships, 19, 775-795. doi:10.1111/j.14756811.2011.01392.x.

Myers, S. A. \& Goodboy, A. K. (2010). Relational maintenance behaviors and communication channel use among adult siblings. North American Journal of Psychology, 12, 103-116.

Ogolsky, B. (2009). Deconstructing the association between relationship maintenance and commitment: Testing two competing models. Personal Relationships, 16, 99-115.

Oswald, D. L. \& Clark, E. M. (2006). How do friendship maintenance behaviors and problem solving styles function at the individual and dyadic levels? Personal Relationships, 13, 333-348.

Öz Soysal, F. S., Uz Baş, A. \& Aysan, F. (2019). The study of reliability and validity of relationship maintenance strategies scale. Electronic Journal of Social Sciences, 18 (70), 710-719.

Ramirez, A. (2008). An examination of the tripartite approach to commitment: An actor partner interdependence model analysis of the effect of relationship maintenance behavior. Journal of Social and Personal Relationships, 25, 943-965.

Roscoe, B., Cavanaugh, L. E. \& Kennedy, D. R. (1988). Dating infidelity: Behaviors, reasons, and consequences. Adolescence, 23, 35-43.

Schutz, W. (1966). The interpersonal underworld. Palo Alto, CA: Science and Behavior Books.

Schermelleh-Engel. K \& Moosbrugger. H. (2003). Evaluating the fit of structural equation models: Tests of significant descriptive goodness of-fit measures. Methods of Research Online, 8(2), 23-74.

Simon, V. A., Kobielski, S. J. \& Martin, S. (2008). Conflict beliefs, goals, and behavior in 
romantic relationships during late adolescence, Journal of Youth and Adolescence, 37, 324-335. doi: 10.1007=s10964-007-9264-5.

Stafford, L. (1994). Measuring relationship maintenance behaviors: Critique and development of the revised relationship maintenance behavior scale, Journal of Social and Personal Relationships28(2), 278-303. doi: 10.1177/0265407510378125.

Stafford, L., Dainton, M. \& Haas, S. (2000). Measuring routine and strategic relational maintenance: Scale revision, sex versus gender roles, and the prediction of relational characteristics. Communication Monographs, 67, 306-323.

Tabachnick, B. G \& Fidell, L.S. (2015). Use of multivariable statistics (Translated by: Baloğlu, M.). (pp. 78-82) Ankara: Nobel Yayınc1lı.

Weigel, D. J. \& Ballard-Reisch, D. S. (2008). Relational maintenance, satisfaction, and commitment in marriages: An actor-partner analysis. Journal of Family Communication, 8(3), 212-229. doi: 10.1080/15267430802182522.

Weiser, D. A. \& Weigel, D. J. (2016). Self-efficacy in romantic relationships: direct and indirect effects on relationship maintenance and satisfaction. Personality and Individual Differences, 89, 152- 156. .doi: 10.1016/j.paid.2015.10.013.

Yurdabakan, İ. (2008). Qualifications of measurement tools used in education. Measurement and Assessment in Education (Edit: S.Erkan and M. Gömleksiz). (pp. 38-66). Ankara: Nobel Yayın Dağıtım. 